\title{
Novel low-cost control system for large high-speed corn precision planters
}

\author{
Youqiang Ding ${ }^{1,2}$, Li Yang ${ }^{1,2^{*}}$, Dongxing Zhang ${ }^{1,2}$, Tao Cui ${ }^{1,2}$, Yuhuan $\mathrm{Li}^{1,2}$, \\ Xiangjun Zhong ${ }^{1,2}$, Chunji Xie ${ }^{1,2}$, Zhengliang Ding ${ }^{1,2}$ \\ (1. College of Engineering, China Agricultural University, Beijing 100083, China; \\ 2. Soil-Machine-Plant Key Laboratory of Ministry of Agriculture and Rural Affairs, Beijing 100083, China)
}

\begin{abstract}
Large high-speed corn precision planters can significantly improve seeding efficiency, but at present, there is no mature control technology in China. This study proposed a novel low-cost control system for large high-speed corn precision planters based on multiple technologies such as embedded systems, controller area network (CAN), global positioning system (GPS), and Android development technology. The developed control system provided excellent expansion capabilities of more than 40 planter rows. Functions such as monitoring the planter status, adjusting seed density, calculating planting-area were realized. Field experiments were performed under different GPS frequencies $\left(f_{G P S}\right)$, travel speeds, and seed spacings. Results showed that the working area relative error index $(R E I)$ and $f_{G P S}$ were negatively correlated, and the planter can achieve $0.84 \%$ error under the $f_{G P S}$ of $10 \mathrm{~Hz}$. When the travel speed was $10 \mathrm{~km} / \mathrm{h}$ or $12 \mathrm{~km} / \mathrm{h}$, the average quality index $(Q I)$, miss index $(M I)$, and precision index $(P I)$ were superior to $92.84 \%, 5.80 \%$, and $18.57 \%$ under each seed spacing, respectively, $Q I$ and $M I$ had no significant difference under each seed spacing $(p<0.05)$. For a travel speed of $14 \mathrm{~km} / \mathrm{h}$, indexes values reached the worst level at seed spacing of $20 \mathrm{~cm}$, but still met the requirements of Chinese national standards. The total cost of the control system for a 12-row corn precision planter is only $17.07 \%$ of similar products from abroad. In summary, the developed control system can achieve preferable performance and costs much less than similar products obtained from abroad, which is suitable for promotion in China or other developing countries.
\end{abstract}

Keywords: corn precision planter, control system, Android, CAN bus, GPS

DOI: $10.25165 /$ j.ijabe. 20211402.6053

Citation: Ding Y Q, Yang L, Zhang D X, Cui T, Li Y H, Zhong X J, et al. Novel low-cost control system for large high-speed corn precision planters. Int J Agric \& Biol Eng, 2021; 14(2): 151-158.

\section{Introduction}

According to statistical data from 2017, the current number of people who primarily engage in agricultural production or operation in China is 314.22 million. Compared with data from 2006 and 1996, this number has decreased by $8.6 \%$ and $25.9 \%$, respectively ${ }^{[1,2]}$. With the acceleration of industrialization and urbanization in China, many rural laborers are moving to cities. Meanwhile, the transfers of contracted land and management rights have been increasing, and the level of agricultural technology and equipment has been continuously improving. Under the conditions mentioned above, scale management of land has become the inevitable development trend of modern agriculture.

Large agricultural machinery plays an important role in scale land management; for example, in the seeding stage of corn

Received date: 2020-07-29 Accepted date: 2020-11-10

Biographies: Youqiang Ding, PhD candidate, research interests: precision agriculture and intelligent control for planters, Email: dyq@cau.edu.cn; Dongxing Zhang, Master, Professor, research interests: full mechanization of corn production, Email: zhangdx@cau.edu.cn; Tao Cui, PhD, Associate Professor, research interests: full mechanization of corn production, Email: cuitao@cau.edu.cn; Yuhuan Li, PhD candidate, research interests: corn precision seed meters designing, Email: 18612897966@163.com; Xiangjun Zhong, PhD candidate, research interests: precision agriculture and variable rate seeding technology, Email: xjzhong1004@163.com; Chunji Xie, Master, research interests: seeding detection technology, Email: xiecj@cau.edu.cn; Zhengliang Ding, Master, research interests: intelligent control for corn precision planters, Email: 18339206361@163.com.

*Corresponding author: Li Yang, $\mathrm{PhD}$, research interests: precision agriculture. College of Engineering, China Agricultural University, Beijing 100083, China. Tel: +86-10-62737765, Email: yangli@cau.edu.cn. production, the use of large high-speed corn precision planters has the following advantages: saving labors, reducing the number of machine passes, easing soil compaction, and improving operating efficiency. Despite these advantages, the width of domestic precision planters employed in current corn production is mostly within 1200-3600 $\mathrm{mm}$ and the planters are driven by the ground wheel and gearbox, and slippage between the wheel and ground typically results in poor seeding quality especially under high travel speed $^{[3,4]}$. Therefore, traditional corn planter efficiency is limited by the row number of the planter and the drive mode of seed meters. Similarly, for large high-speed corn precision planters, it will be even harder to provide enough power for the multi-row adopting ground wheel and gearbox. Besides, the failure of control system during operation in large high-speed corn precision planters (e.g., drive motor stop, loss of travel speed signal) will lead to more yield losses than that in traditional planters due to their high speed and wide-scale ${ }^{[5,6]}$. Therefore, solving the two problems mentioned above is the key to realize the high-efficiency potential of large high-speed corn precision planters.

The research on electric or hydraulic drive systems for planters started a few decades ago in the $1980 \mathrm{~s}^{[7]}$. In recent years, many researchers have proposed using electric or hydraulic drive systems combined with seeding monitoring technology to drive seed meters instead of mechanical transmission systems ${ }^{[8-10]}$. Cay et al. ${ }^{[11,12]}$ adopted pulse width modulation and used a proportional-integral-derivative (PID) technique to develop a mechatronics control system for a 4-row corn precision planter, and field experiment results showed that the quality index $(Q I)$ of researched control system was 2.5 percentage points more than that of traditional mechanical drive systems. Ranta et al. ${ }^{[13]}$ found that 
electric drive systems created much better quality and can be twice as efficient as a traditional planter. Moreover, world-class planter manufacturers such as John Deere (USA), Kinze (USA), Horsch (Germany), Precision Planting (USA), and Maschio (Italy) have already developed advanced electric drive systems and varieties of planters from small to large scale. Edge techniques in plant field including variable-rate seeding, curve compensation, and automation section control are integrated into their control system. These planter products can accommodate operating speeds as high as $16 \mathrm{~km} / \mathrm{h}$, and provide high control accuracy and reliability. However, these high-performance planters are very expensive and difficult to implement in China or other developing countries.

In China, a large amount of research has been conducted on electric drive systems for corn precision planters. Zhang et al. ${ }^{[14]}$ developed a control system for a single-row corn precision planter that employed a brushless direct current motor (BLDCM) to drive seed meters and radar to acquire the travel speed of the planter, and got a $92.40 \% Q I$ value. Yang et al. ${ }^{[15]}$ utilized optical encoders to measure the planter's speed and employed step motors to drive corn precision seed meters along its circumference. Field experiment results showed that the system exhibited superior performance with an average $4.70 \%$ increase of $Q I$ and $3.54 \%$ decrease of miss index $(M I)$ as compared with a traditional planter, especially in high travel speed cases. Ding et al. ${ }^{[16]}$ employed a global positioning system (GPS) receiver to acquire planter travel speed and BLDCM to drive the seed meter. The control system was designed with features of parameters invisible in real-time and can be adjusted during operation. Research on electric drive systems for corn precision planters has garnered more and more interest and attention in recent years in China, but most of the developed control systems are still in the laboratory testing stage and have yet to be commercialized. Furthermore, most researches still focus on small-scale corn precision planters, which do not meet the needs of large agricultural machinery for the trend of large-scale land management.

The primary purpose of this study was to solve the problem of the high cost of imported planter products and domestic situation of lacking control systems for large high-speed corn precision planters by (1) proposing a low-cost control system for a large high-speed corn precision planter based on multiple intersectional technologies, such as CANopen master-slave control net, GPS, embedded technology, and Android development technology; (2) designing a hardware circuit of the central controller, which is kernel parts of the whole control system; (3) developing embedded software and an Android seeding application; (4) conducting field experiments to verify the performance of the proposed control system.

\section{Materials and methods}

\subsection{System overall design}

The proposed control system was mainly composed of an Android tablet (Huawei Technologies Co., Ltd., China), GPS receiver (Beitian Communication Co., Ltd., China), central controller, and 12-row drive system (RDS). As shown in Figure 1, the Android tablet was utilized as a human-computer interaction device, and the GPS receiver was installed on the tractor cab and used to measure the travel speed of the planter. Also, the core component of the central controller is an STM32F105RCT6

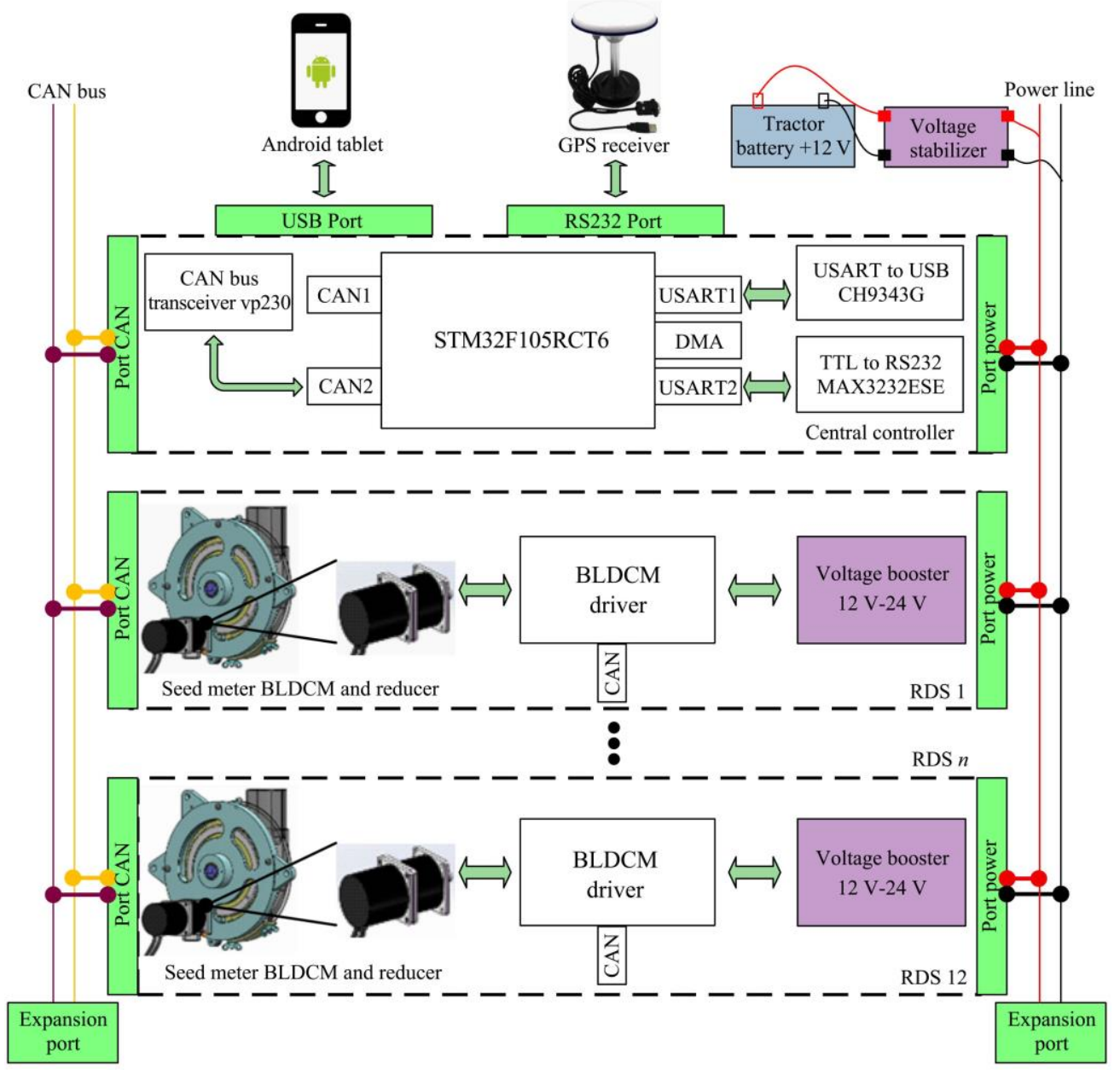

Figure 1 Components of the control system 
microprocessor (STMicroelectronics Co., Ltd., Switzerland), which is responsible for collecting and distributing data of the whole control system with its $72 \mathrm{MHz}$ high operation frequency. The RDS was used as planting execution with units consisting of a voltage booster (Eveps Technology Co., Ltd., China), a BLDCM driver (Aikeli Automation Technology Co., Ltd., China), a BLDCM (Times Brilliant Electrical Co., Ltd., China), a reducer (Ruide Transmission Technology Co., Ltd., China) and a corn precision seed meter (the Corn Mechanization Lab of China Agricultural University, China). Some important components and parameters of the control system were listed in Table 1 . The CANopen high-level application layer protocol based on CAN bus was utilized between the central controller and the RDS; this kind of communication provides good extensibility for the control system and makes increasing or decreasing the RDS more convenient. Most components of the control system are produced by domestic manufacturers or are available on the market in China; the total cost of the control system for a 12-row corn precision planter is only $\$ 5120.40$, details of which are shown in Table 2. This cost is significantly lower than similar products imported from abroad; for example, Precision Planting's popular planter control products, such as Vdrive, Vset, Delta force, Wavevisions and 20/20, cost more than $\$ 30000$ for a 12-row precision corn planter. Furthermore, the Android tablet used in the control system also can be replaced by the operator's smart Android phone by logging into the software market on the internet and downloading the Android seeding application developed and uploaded by the authors. Further savings can be achieved in this way.

Table 1 Important components and parameters of the control system

\begin{tabular}{cc}
\hline Components & Key parameters \\
\hline GPS receiver & Rated voltage: $5 \mathrm{~V}$; Operation temperature: $-40^{\circ} \mathrm{C}-85^{\circ} \mathrm{C} ;$ \\
Speed accuracy: $0.1 \mathrm{~m} / \mathrm{s}$
\end{tabular}

Table 2 Total cost (unit: US dollar) of the control system for a 12-row corn precision planter

\begin{tabular}{ccccc}
\hline Component name & Unit price $^{\mathrm{a}}$ & Quantity & Cost $^{\mathrm{a}}$ & Total cost $^{\mathrm{a}}$ \\
\hline GPS receiver & 37.43 & 1 & 37.43 & \\
Android tablet & 169.47 & 1 & 169.47 & \\
Central controller (self-design) & 98.86 & 1 & 98.86 & \\
Voltage booster & 6.50 & 12 & 78.00 & \multirow{2}{*}{5120.40} \\
BLDCM driver & 56.49 & 12 & 677.88 & \\
BLDCM & 12.71 & 12 & 152.52 & \\
Reducer & 43.07 & 12 & 516.84 & \\
Seed meter & 282.45 & 12 & 3389.4 & \\
\hline
\end{tabular}

Note: ${ }^{a}$ The price was confirmed on June 6,2020 , and the rate of US dollars to $\mathrm{RMB}$ is 7.0808 .

\subsection{Hardware design of the central controller}

The central controller as the core part of the control system is illustrated in Figure 2. The integrated circuit and printed circuit board were designed by Altium designer software 20.0.2 (Altium Ltd. USA). As shown in Figure 2c, the printed circuit board (PCB) of the central controller primarily consists of an STM32F105RCT6 microprocessor as the information computing center, power circuit to obtain stable power supply, universal synchronous asynchronous receiver (USART) to universal serial bus (USB) conversion circuit based on the CH9343 chip (Qinheng Microelectronics Co., Ltd., China) to communicate with an Android tablet, recommended standard 232 (RS232) circuit based on MAX3232ESE chip (Maxim Integrated, Inc., USA) to receive a message from GPS receiver, and CAN bus transceiver circuit based on VP230 chip (Texas Instruments Inc., USA) to communicate with RDS. Furthermore, an Acrylonitrile Butadiene Styrene (ABS) plastic container with waterproof and dustproof features was used to package the PCB to improve the applicability and practicability for field operating conditions. Two LEDs were mounted on the surface of the plastic container. The Android tablet connection status LED will light up once the Android tablet is connected to the central controller, and GPS signal transmission status LED will flash at a frequency of receiving GPS messages. The central controller was installed in the cab of the tractor; hence, the LEDs can be observed visually by the operator, and immediate measures can be taken in the event of communication failure.

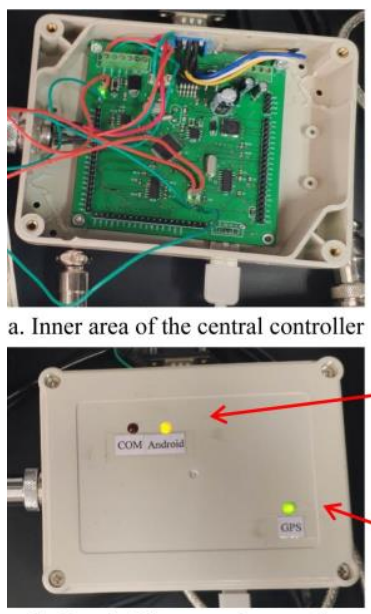

b. Panel of the central controller

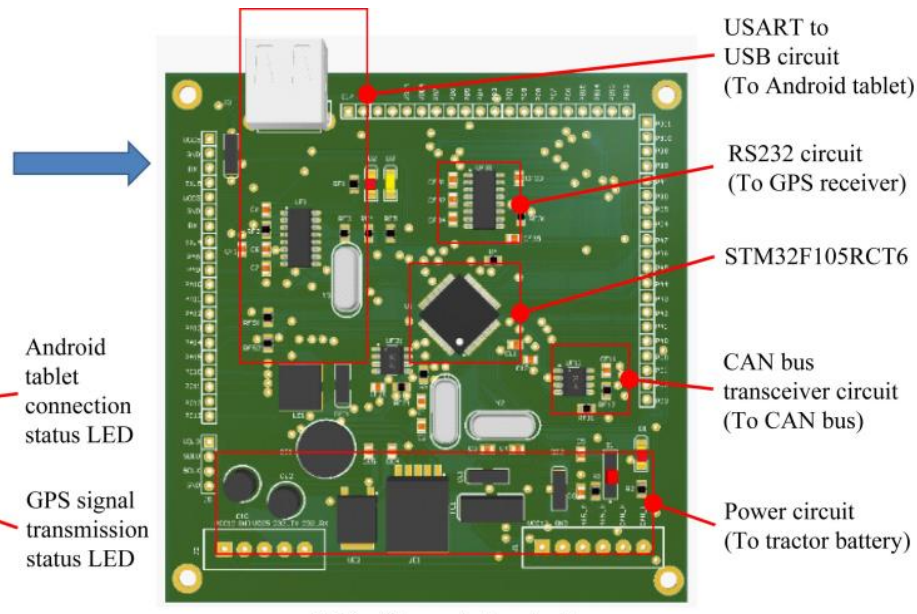

c. PCB of the central controller

Figure 2 Hardware circuit design of the central controller

\subsection{Establishment of the speed adjusting model for BLDCM}

To make the actual seed spacing consistent with the target seed spacing, the parameters of the seed meter, such as reducer transmission ratio, hole number of the disk, travel speed of the planter, and BLDCM rotation speed should be adapted to each other in real-time. The mathematical relationship between different parameters is as follows:

$$
n=5000 v r_{g} / 3 H s
$$

where, $n$ is the rotation speed of the BLDCM, r/min; $v$ is the travel speed of the planter, $\mathrm{km} / \mathrm{h} ; r_{g}$ is the transmission ratio of the 
reducer; $H$ is the hole number of the seed meter's disk; $s$ is the target seed spacing, cm. $r_{g}, H$ and $s$ are input by the operator through an Android tablet, and $v$ is acquired from the GPS receiver (the specific method can be referred to the author's previous research $^{[16]}$ ).

\subsection{Establishment of the working area mathematical model}

The working area $(S)$ of the planter can be calculated in real-time based on the mathematical model presented below. As shown in Figure 3a, the basic approach is to take the integral of velocity over time; the seeding distance can be described with the area enclosed by the $t$-axis, $v$-axis, and the speed curves $v(t)$. Then, the seeding distance is multiplied by the width of the planter $(L)$. However, the continuous curve $v(t)$ does not exist in the computer program; when the planter works in the field, the GPS receiver will acquire the travel speed with a fixed $f_{G P S}$, so $v(t)$ will be discretized and seeding distance will be quantized as Figure $3 \mathrm{~b}$. Finally, the working area can be calculated by Equations (2) and (3). Also, the seeding signal is used as a switch for integration operations as illustrated in Figure $3 \mathrm{a}$ (red areas indicate that no seeds are falling and the integration algorithm is off). For example, BLDCM will be turned off under some conditions, such as the planter reaching the boundary of the field and needing to take turning operations; at this time, the integration algorithm is off.

$$
\begin{gathered}
\Delta t=1 / f_{\text {GPS }} \\
S=\frac{L}{2} \sum_{t=0}^{n}\left(v_{t}+v_{t+\Delta t}\right) \Delta t
\end{gathered}
$$

where, $\Delta t$ is the GPS signal interval, $\mathrm{s} ; v_{t}$ is the travel speed of the planter at time $t, \mathrm{~m} / \mathrm{s} ; \quad n$ is the GPS sampling number from starting.

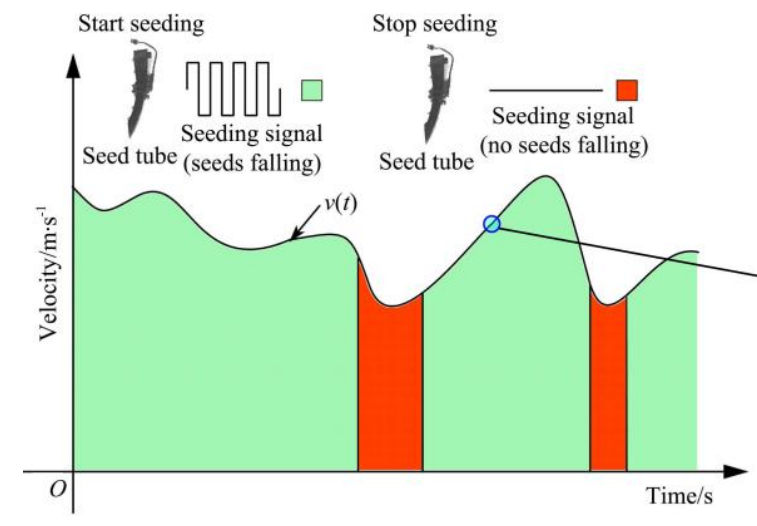

a. Seeding distance integral calculation model and principle

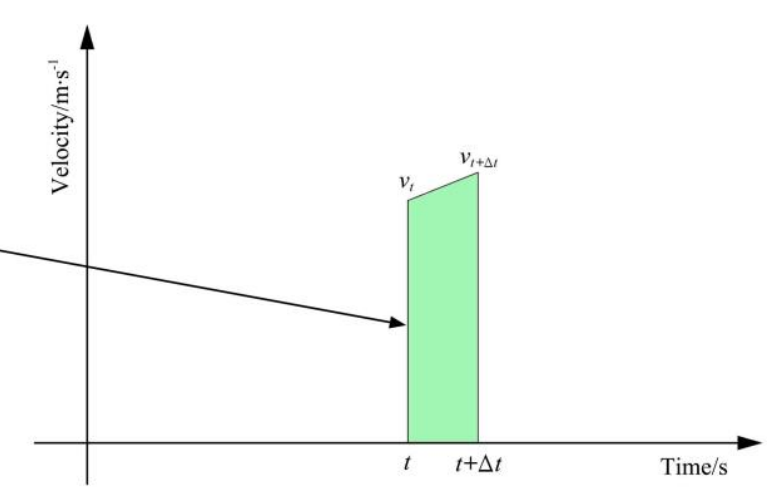

b. Seeding distance element

Figure 3 Integral model of seeding distance

\subsection{Software design of the control system}

2.5.1 Function of planter row status monitoring based on CANopen protocol

The BLDCM driver has a built-in CiA DSP402 motion control protocol, which is a sub-protocol of the CANopen high-level application layer protocol based on the CAN bus. In this system, the central controller and BLDCM drivers are used as the master node and slave node respectively. The master node mainly manages the CANopen net through net management messages (NMT), service data objects (SDO), and process data objects (PDO), as well as the control and monitoring of each slave node. There are three important functions realized based on NMT, SDO, and PDO.

(1) "Hot-swap" of the RDS. As shown in Figure 4a, according to the DSP402 motion control protocol, an NMT boot-up message with an exclusive ID number will be sent to the CANopen net at the moment a new RDS is connected (new slave node online). After receiving the boot-up message, the master node is programmed to send initialization commands to the CANopen net according to the ID number to complete the initialization of the new slave node. As such, there is no need to restart the control system when adding or replacing an RDS.

(2) BLDCM rotation speed closed-loop control. The SDO message is mainly used to adjust the BLDCM's rotation speed in this control system. As illustrated in Figure 4b, during planting, the master node will constantly access the object dictionary related to the rotation speed of the BLDCM driver by sending an SDO message. In addition, the BLDCM driver also measures the actual rotation speed of the BLDCM based on its hall signal and employs the PID algorithm to achieve more accurate closed-loop control.

(3) RDS status monitoring. RDS possible faults during the planting operation mainly include BLDCM spin down, insufficient voltage supply, and being offline from the CANopen net, etc. This study solved this problem based on the DSP402 motion control protocol, as shown in Figure 4c. The process proceeds as follows: 1) the master node transfers the CANopen state machine of the BLDCM driver into the operational state by sending an NMT message, and then PDO will be activated. 2) configure PDO mapping parameters as real-time actual rotation speed, status word. 3) Configure the transmission frequency of PDO messages. After the above settings are completed, the master node will determine whether the RDS is in error status by parsing the PDO message; if so, an alarm message will be sent to the Android tablet.

\subsubsection{Workflow of the control system}

As shown in Figure 5, the operator first inputs planting parameters such as transmission ratio, hole number, and target seed spacing through the Android seeding application installed on the Android tablet after the control system is powered on. Meanwhile, the central controller completes the initialization of each slave node and configures PDO parameters, and then travel speed, location, and working area of the planter will be parsed or calculated after the GPS message is received from the RS232 interface, and all the information will be sent back to the Android tablet through a USB cable. Subsequently, the BLDCM rotation speed is calculated based on planting parameters inputted and the planter travel speed according to Equation (1). Next, the central controller will adjust BLDCM rotation speed in real-time as mentioned in Equation (2). In the event of failures coming to the RDS, the central controller will determine errors from the state word as mentioned in Equation (3), and then an alarm will be sent to Android tablet through the USB cable to inform the operator to stop the planter and check (User interaction layer of the Android seeding application in Figure 
5 shows an example of the first RDS break down). So it can be control and real-time planter status monitoring. concluded that the control system realized functions of seeding

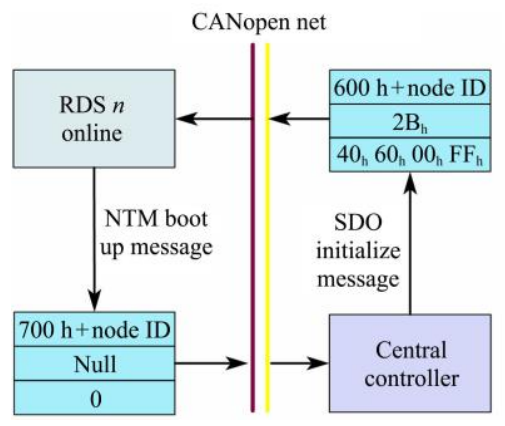

a. Message flow of "Hot-swap" of the RDS

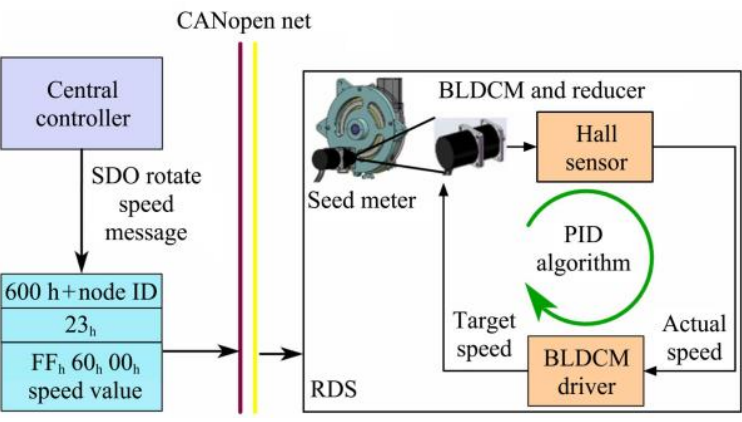

b. Message flow of BLDCM rotation speed adjusting

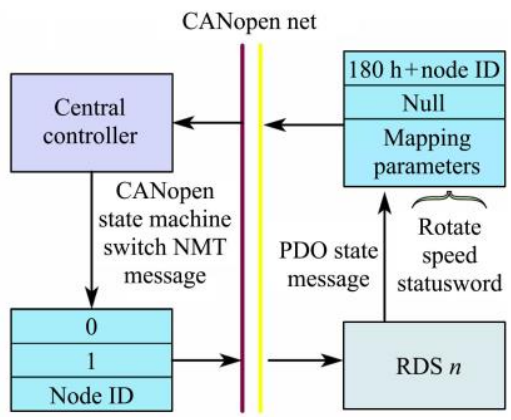

c. Message flow of RDS status monitoring

Figure 4 CANopen communication mode in the control system

Android seeding application

User interaction layer

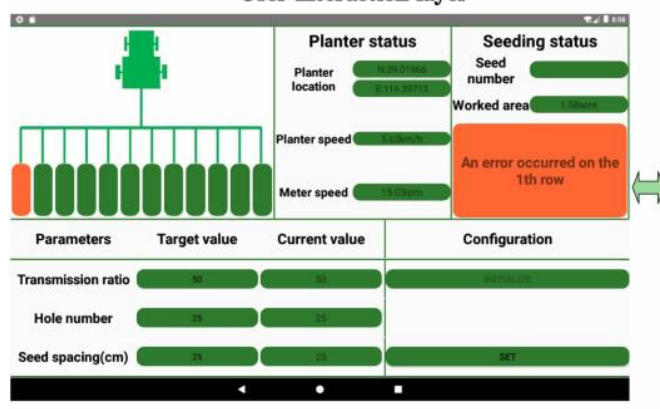

GPS receiver
Central controller software

Figure 5 Program flowchart of the whole control system

\subsubsection{CANopen net load calculation and capacity analysis}

In practical application, the load rate of the CAN bus is generally required not to exceed $30 \%$ to prevent the system from bus congestion and error frames. Thus, the capacity analysis was conducted in this study to calculate the maximum expansion capacity of the control system. According to 2.5.2, the system can be divided into three stages during operation as follows. (a) Switch the control system on, slave nodes go online, and send the node NMT to boot up messages and NMT heartbeat message to CANopen net. (b) The central controller sends the slave node SDO initialization message to the CANopen net. Meanwhile, the NMT heartbeat message is in activated status at this stage. (c) The central controller sends the SDO BLDCM rotation speed adjusting instruments in a fixed frequency; additionally, PDO and NMT heartbeat messages are in activated status at this stage. It can be found that the data volume reaches the maximum at (c) stage, which can be calculated by the following equation:

$$
\begin{aligned}
& \mu=N \cdot \sum_{1}^{n} \mu_{i} \\
& \mu_{i}=f_{i} \cdot l_{i} / B \times 100 \%
\end{aligned}
$$

where, $\mu$ is the total CAN bus load rate of the control system, $\%$; $N$ is the number of the planter rows; $n$ is the number of different message types; $\mu_{i}$ is the CAN bus load rate of the $i$-th message, \%; $f_{i}$ is the sending frequency of the $i$-th message, $\mathrm{Hz} ; l_{i}$ is the bit length of the $i$-th message, bit; and $B$ is the baud rate of the CAN bus, bit/s. Figure 6 shows the structure of the CANopen message frame and bit length of different fields. It can be determined that except for the data field, the bit length of other fields for different messages is the same, so $l_{i}$ can be expressed by the following equation:

$$
l_{i}=44+l_{\text {data_field }_{-}}+3
$$

where, 44 is the total bit length of a message except data field; $l_{\text {data_field }}$ is the bit length of the data field, and there are 3 bits intervals between different messages according to the CANopen protocol. As shown in Table 3, the $f_{i}$ of NMT, SDO, and PDO at the (c) stage were set to $5 \mathrm{~Hz}, 20 \mathrm{~Hz}$, and $10 \mathrm{~Hz}$, respectively, the $B$ of the CAN bus was configured to $500 \mathrm{~kb} / \mathrm{s}$. Finally, the $\mu$ of the control system was calculated as $8.652 \%$ based on Equations (4) and (5), which was much less than the $30 \%$ threshold. Besides, it can be also concluded that the maximum planter row expansion number is 41 , which meets the requirements of different scale corn precision planters. Furthermore, the control system can also be employed on planters with rows more than 41 by increasing the $B$ or decreasing the $f_{i}$ of NMT, SDO, and PDO within the allowable range.

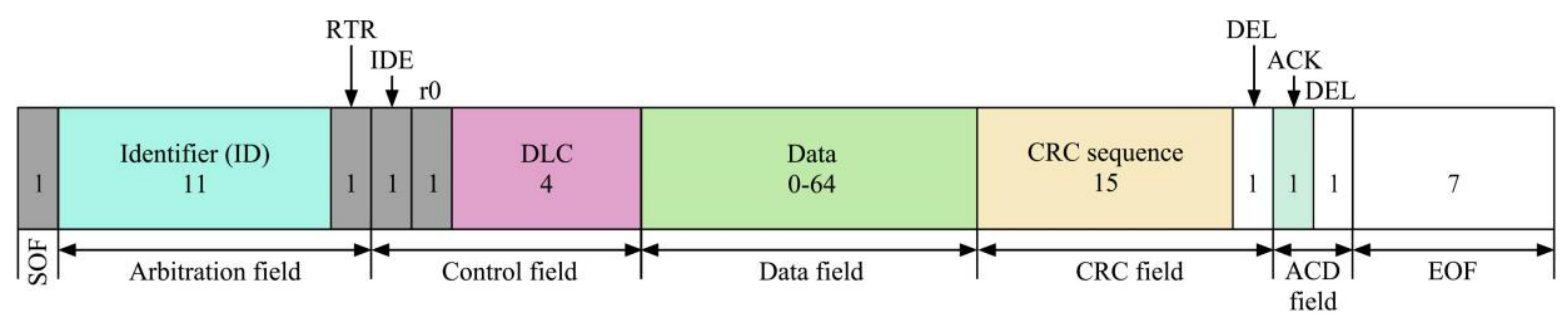

Figure 6 Structure of CANopen message frame and bit length of different fields 
Table 3 CAN bus attribute values of the control system

\begin{tabular}{|c|c|c|c|c|c|c|}
\hline Message type & $l_{\text {data_field }} / \mathrm{bit}$ & $f_{i} / \mathrm{Hz}$ & $\mu_{i} / \%$ & B/bit $\cdot \mathrm{s}^{-1}$ & $N$ & $\mu / \%$ \\
\hline NMT & 8 & 5 & 0.055 & $500 \mathrm{k}$ & 12 & 8.652 \\
\hline SDO & 64 & 20 & 0.444 & & & \\
\hline PDO & 64 & 10 & 0.222 & & & \\
\hline
\end{tabular}

\subsection{Field experiment}

\subsubsection{Experiment conditions}

The field experiment was performed on September 27, 2019, at the test base of Lovol Arbos Agricultural Machinery Division in Zhucheng, Shandong Province, China. The whole control system was mounted on a Lovol Arbos 2BTQF12A 12-row corn no-tillage precision planter (Figure 7). The variety of corn seeds utilized in the experiment was Zhengdan 958, which had a 1000-seed weight of $332 \mathrm{~g}$ and a moisture content of $13 \%$, not grade. The air pressure range of each planter row was maintained within 3.5$4.5 \mathrm{kPa}$. Before the experiment, the field soil was shallow-spinned with a depth of $10 \mathrm{~cm}$ and dried for $2 \mathrm{~d}$ to obtain good implantation conditions for corn seeds.

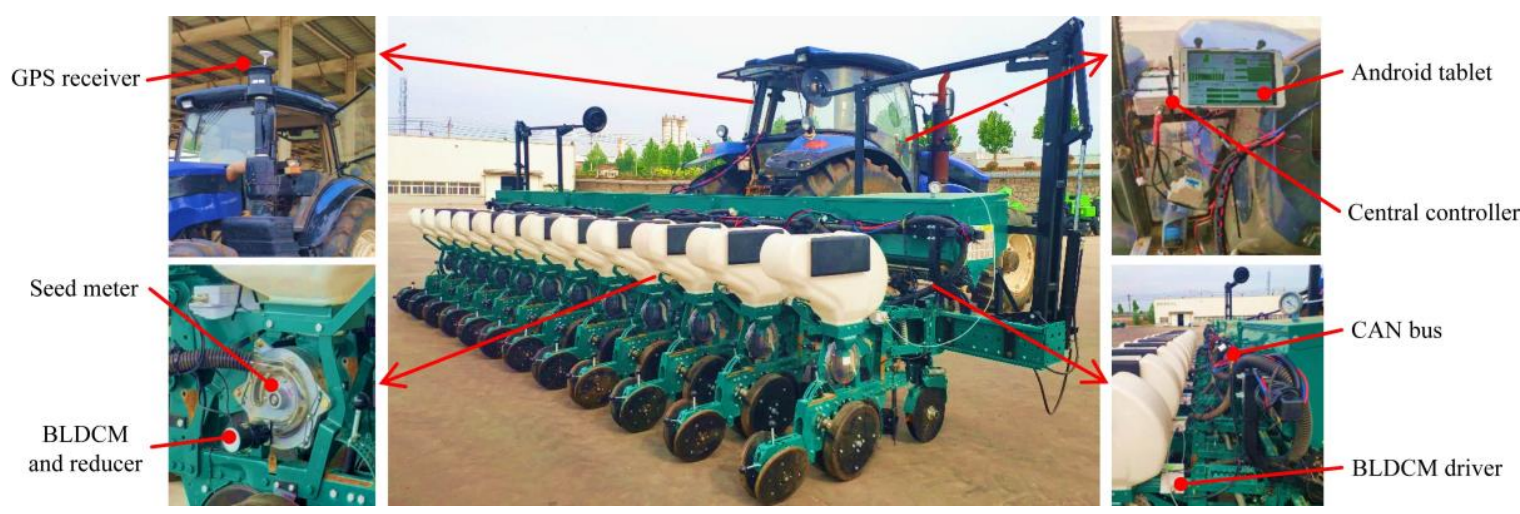

Figure 7 Lovol Arbos 2BTQF12A 12-row corn no-tillage precision planter and layout of the control system

\subsubsection{Experiment arrangement}

Working area statistical experiments were performed under three different GPS frequencies $(1 \mathrm{~Hz}, 5 \mathrm{~Hz}$, and $10 \mathrm{~Hz})$, and each factor level was repeated three times. Relative error index (REI) was selected to evaluate the accuracy of the working area mathematical model and can be obtained by the following equation:

$$
R E I=\frac{|A A V-M A V|}{A A V} \times 100 \%
$$

where, $A A V$ is the actual working area value, $\mathrm{hm}^{2} ; M A V$ is the measured working area value, $\mathrm{hm}^{2} ; R E I$ is the relative error index, $\%$.

Seeding performance experiments were conducted under three different traveling speeds $(10 \mathrm{~km} / \mathrm{h}, 12 \mathrm{~km} / \mathrm{h}$, and $14 \mathrm{~km} / \mathrm{h})$ and three different seed spacings $(20 \mathrm{~cm}, 22 \mathrm{~cm}$, and $25 \mathrm{~cm}$ ), and each factor level was repeated three times. After the experiment was completed, three regions were randomly selected to collect 250 seed spacings, respectively. $Q I, M I$, and precision index $(P I)$ were used as indicators to evaluate the performance of the control system and can be calculated according to ISO 7256/1-1984(E) Standard:

$$
\begin{gathered}
D I=\frac{n_{1}}{N} \times 100 \% \\
M I=\frac{n_{2}}{N} \times 100 \% \\
Q I=100 \%-(D I+M I) \\
P I=\frac{S_{d}}{s} \times 100 \%
\end{gathered}
$$

where, $D I$ is the multiple index, $\% ; n_{1}$ is the number of seed spacing from 0 to $0.5 s ; n_{2}$ is the number of seed spacing from $1.5 s$ to $+\infty ; N$ is the total number of seed spacing; $S_{d}$ is the standard deviation of seed spacing.

\section{Results and discussion}

The working area statistical results are shown in Table 4, and the actual area of the experiment field was measured by a long tapeline. From Table 4, a conclusion can be drawn that REI and $f_{G P S}$ are negatively correlated. This may be because under the same measurement error condition, the faster the frequency of GPS receiver signal updates, the better the fit of measured speed value with the actual speed curve $v(t)$ is. The maximum update frequency of the GPS receiver employed in this study is $10 \mathrm{~Hz}$, with this condition, REI between the average measured area and the actual area is $0.84 \%$, which can satisfy the accuracy requirements of the operator and can be used as the basis for charging seeding service.

Table 3 REI between average measured area and actual area under different $f_{G P S}$

\begin{tabular}{cccc}
\hline Actual area $/ \mathrm{hm}^{2}$ & $f_{G P S} / \mathrm{Hz}$ & Average measured area $/ \mathrm{hm}^{2}$ & $R E I / \%$ \\
\hline \multirow{3}{*}{4.3342} & 1 & 4.2573 & 1.77 \\
& 5 & 4.4070 & 1.68 \\
& 10 & 4.2978 & 0.84 \\
\hline
\end{tabular}

The statistical results of seeding performance experiments are shown in Table 5. A two-factor analysis of variance was performed using SPSS 17.0 (SPSS Inc., USA) to determine whether the difference between different factor levels was significant at a $95 \%$ confidence level. The analysis results were plotted in Figure 8. From these results, the following trends were found:

(1) Influence of different factors on $Q I$ and $M I$.

As Figures $8 \mathrm{a}$ and $8 \mathrm{~b}$ shown, when the traveling speed was $10 \mathrm{~km} / \mathrm{h}$ or $12 \mathrm{~km} / \mathrm{h}$, there was no significant difference in average $Q I$ and average $M I$ under different seed spacing conditions, and all of the average $Q I$ and average $M I$ were above $92.84 \%$ and below 
$5.80 \%$, respectively. However, when the traveling speed reached $14 \mathrm{~km} / \mathrm{h}, Q I$ and $M I$ showed a significant difference between $20 \mathrm{~cm}$ seed spacing and the other two seed spacings, and the $Q I$ declined under all seed spacing conditions compared with $10 \mathrm{~km} / \mathrm{h}$ and $12 \mathrm{~km} / \mathrm{h}$, which reached the lowest value of $84.11 \%$ under $20 \mathrm{~cm}$ seed spacing. However, the effect on the $M I$ was just the opposite, which increased and reached the highest value of $11.97 \%$ under the $20 \mathrm{~cm}$ seed spacing.

(2) Influence of different factors on PI.

As shown in Figure 8c, when the travel speed was $10 \mathrm{~km} / \mathrm{h}$, there was no significant difference on average PI between the $20 \mathrm{~cm}$ and the $22 \mathrm{~cm}$ seed spacing, but there was a significant difference between these two seed spacings and the $25 \mathrm{~cm}$ seed spacing. Moreover, the PI reached the lowest value of $15.03 \%$ under the condition of the $25 \mathrm{~cm}$ seed spacing. When the travel speed reached $12 \mathrm{~km} / \mathrm{h}$, there was no significant difference under all seed spacing conditions. However, there was a significant difference in average $P I$ between $20 \mathrm{~cm}$ and the other two seed spacings under the traveling speed of $14 \mathrm{~km} / \mathrm{h}$, and the average $P I$ under each seed spacing condition reached the maximum value at three speeds, of which the average $P I$ under the $20 \mathrm{~cm}$ seed spacing was the largest, at $24.24 \%$.

Table $5 Q I, M I$, and $P I$ values (mean \pm standard deviation) of seeding performance experiments under different factors and levels

\begin{tabular}{ccccc}
\hline $\begin{array}{c}\text { Travel } \\
\text { speed } / \mathrm{km} \cdot \mathrm{h}^{-1}\end{array}$ & $\begin{array}{c}\text { Seed } \\
\text { spacing/cm }\end{array}$ & $Q I / \%$ & $M I / \%$ & $P I / \%$ \\
\hline \multirow{2}{*}{10} & 20 & $93.02 \pm 2.33$ & $5.80 \pm 1.84$ & $17.66 \pm 0.87$ \\
& 22 & $92.84 \pm 1.85$ & $3.16 \pm 0.51$ & $18.44 \pm 1.65$ \\
& 25 & $95.90 \pm 1.48$ & $3.26 \pm 1.02$ & $15.03 \pm 0.73$ \\
\hline \multirow{2}{*}{12} & 20 & $93.62 \pm 1.01$ & $5.01 \pm 1.12$ & $18.57 \pm 0.83$ \\
& 22 & $95.39 \pm 3.46$ & $2.44 \pm 1.46$ & $17.22 \pm 1.67$ \\
& 25 & $94.25 \pm 2.24$ & $4.76 \pm 0.35$ & $16.77 \pm 0.17$ \\
\hline \multirow{2}{*}{14} & 20 & $84.11 \pm 4.33$ & $11.97 \pm 4.34$ & $24.24 \pm 3.03$ \\
& 22 & $91.58 \pm 2.35$ & $6.36 \pm 1.05$ & $18.75 \pm 0.30$ \\
& 25 & $89.66 \pm 2.98$ & $7.24 \pm 2.77$ & $20.08 \pm 1.87$ \\
\hline
\end{tabular}
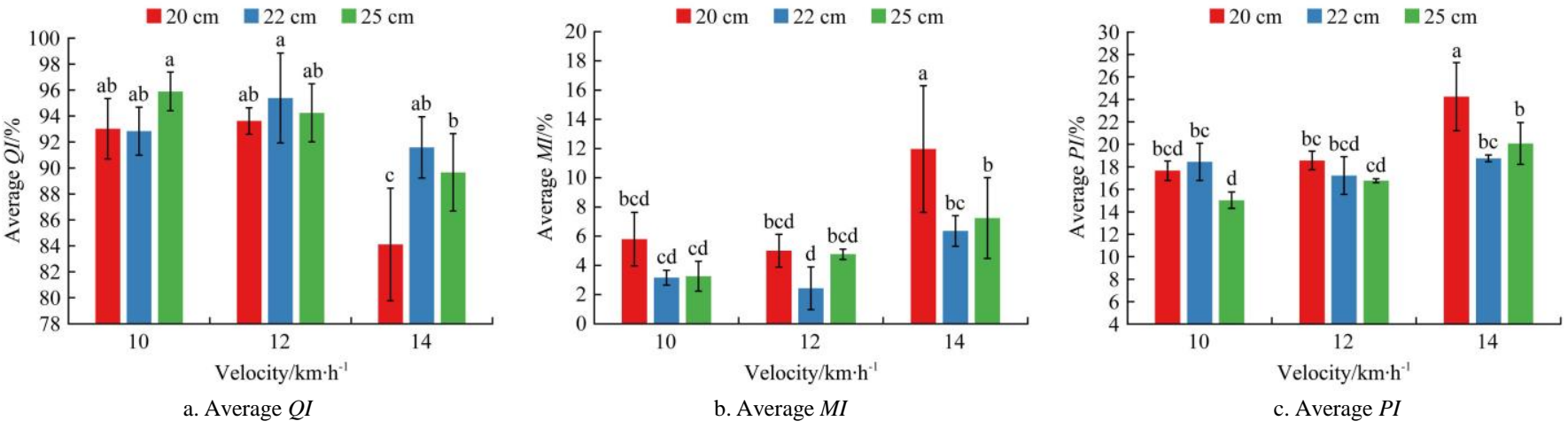

Note: Columns labeled with the same letters are not significantly different $(p<0.05)$.

Figure 8 Performance of the 12-row planter under three different travel speeds and three different seed spacing

In conclusion, the increase of the seed spacing was basically beneficial to experiment indicators, which may be because at the same traveling speed, the bigger the seed spacing, the slower the BLDCM, which in turn improved seeds filling and carrying capacity of the seed discs. Also, the initial velocity of seed escaping from the seed discs can be reduced, and the collision of seeds in the seed tube and the jumping on the seedbed can be diminished. Finally, the indicators of seeding quality such as $Q I$, $M I$, and $P I$ can be improved. However, increasing traveling speed had a negative impact on the experiment indicators, especially when the speed reached $14 \mathrm{~km} / \mathrm{h}$, which may be because the increased impact of vibration intensified in the direction perpendicular to the ground during high-speed operation, resulting in a decrease in the seed filling efficiency or seeds falling out of the seed disc hole again after the seed filling was completed. Conversely, the uncertainty of the movement of seeds in the seed tube will increase with the increase of traveling speed, and it is difficult for the seed flow to fall into the seedbed in an orderly manner, which eventually leads to a decline in the seeding quality. Currently, the world's leading agricultural machinery companies mainly solve the problem of speed by developing specialized seed delivery devices, such as Kinze's True Speed, John Deere's ExactEmerge, and Precision Planting's SpeedTube ${ }^{[17-19]}$, which have all dramatically improve seed flow order, and in turn improve the high-speed operation performance of the planter. Finally, for the developed control system, when the travel speed was $10 \mathrm{~km} / \mathrm{h}$ or $12 \mathrm{~km} / \mathrm{h}$, the average $Q I$ of different seed spacing was no less than $92.84 \%$, the average $M I$ was no more than $5.80 \%$, and the average $P I$ was superior to $18.57 \%$. All the indexes are better than the Chinese national standard ( $Q I \geq 80 \%, M I \leq 15 \%, P I \leq 30 \%)$ requirements for precision planters. Under the traveling speed of $14 \mathrm{~km} / \mathrm{h}$, indexes were the worst at the $20 \mathrm{~cm}$ seed spacing, with an average $Q I$ of $84.11 \%$, an average $M I$ of $11.97 \%$, and an average $P I$ of $24.24 \%$, which still met the Chinese national standard requirements. Therefore, the developed novel low-cost control system performs satisfactorily and can achieve better performance at the recommended traveling speed of no more than $12 \mathrm{~km} / \mathrm{h}$. To achieve a higher traveling speed to further improve operating efficiency, it is recommended to adopt a seed delivery device mentioned above.

\section{Conclusions}

In this study, a novel low-cost control system applied to large high-speed corn precision planters was developed, and field experiments were conducted by Lovol Arbos 2BTQF12A 12-row corn no-till precision planter. The main research conclusions are as follows:

(1) The system adopts CAN bus and CANopen master-slave control net with good expansion ability, uses a GPS receiver to measure the travel speed of the planter and an Android tablet as a human-computer interactive device. Functions such as monitoring the planter travel speed, adjusting seed density, detecting failures of planter rows were realized through the control system.

(2) Field experiment results showed that the proposed working area statistics function can achieve $0.84 \%$ error under an $f_{G P S}$ of 
$10 \mathrm{~Hz}$. When the travel speed was $10 \mathrm{~km} / \mathrm{h}$ or $12 \mathrm{~km} / \mathrm{h}$, the average $Q I, M I$, and $P I$ were superior to $92.84 \%, 5.80 \%$, and $18.57 \%$, respectively, which was better than the Chinese national standard requirements for precision planters. Under the travel speed of $14 \mathrm{~km} / \mathrm{h}$, the average $Q I, M I$, and $P I$ reached the worst value at the $20 \mathrm{~cm}$ seed spacing with $84.11 \%, 11.97 \%, 24.24 \%$, respectively, but still met the Chinese national standard requirements.

(3) Most of the components manufacturers utilized in the developed control system are located in China, and the total cost of the control system for a 12 -row corn precision planter is $\$ 5120.40$, only $17.07 \%$ of similar products from abroad, which is suitable for China's national conditions and can be easily implemented in the developing countries.

\section{Acknowledgements}

This study was supported by the National Key Research and Development Program of China (Grant No. 2017YFD0700703), the National Natural Science Foundation of China (Grant No. 51575515), and the National Industry System of Corn Technology of China (CARS-02).

\section{[References]}

[1] Main data bulletin of the third national agricultural census (No. 5). China National Bureau of Statistics, 2017. (in Chinese)

[2] High-quality development plan for new agricultural management and service entities (2020-2022). Ministry of Agriculture and Rural Affairs of the People's Republic of China, 2020. (in Chinese)

[3] He X, Cui T, Zhang D X, Wei J, Wang M, Yu Y, et al. Development of an electric-driven control system for a precision planter based on a closed-loop PID algorithm. Comput Electron Agr, 2017; 136: 184-192.

[4] Yang S, Wang X, Gao Y, Zhao X, Dou H, Zhao C. Design and experiment of motor driving bus control system for corn vacuum seed meter. Transactions of the CSAM, 2019; 50(2): 57-67. (in Chinese)

[5] Qi J T, Jia H L, Li Y, Yu H B, Liu X H, Lan Y B, et al. Design and test of fault monitoring system for corn precision planter. Int J Agric \& Biol Eng, 2015; 8(6): 13-19.
[6] Lu C, Fu W, Zhao C, Mei H, Meng Z, Dong J, et al. Design and experiment on real-time monitoring system of wheat seeding. Transactions of the CSAE, 2017; 33(2): 32-40. (in Chinese)

[7] Chaney P P, Parish R L, Sistler F E. Automatic control system for a sugarcane planter. Appl Eng Agric, 1986; 2(2): 51-54.

[8] Iacomi C, Popescu O. A new concept for seed precision planting. Agriculture and Agricultural Science Procedia, 2015; 6: 38-43.

[9] Kamgar S, Eslami M J. Design, development and evaluation of a mechatronic transmission system for upgrading performance of a row crop planter. Proceeding of the 2012 ASABE Annual International Meeting, Dallas-USA, 2012

[10] Miller E A, Rascon J, Koller A, Porter W M, Taylor R K, Raun W R, et al Evaluation of corn seed vacuum metering systems. Proceeding of the 2012 ASABE Annual International Meeting, Dallas-USA, 2012; 1 : 815-825.

[11] Cay A, Kocabiyik H, May S. Development of an electro-mechanic control system for seed-metering unit of single seed corn planters Part I: Design and laboratory simulation. Comput Electron Agr, 2018; 144: 71-79.

[12] Cay A, Kocabiyik H, May S. Development of an electro-mechanic control system for seed-metering unit of single seed corn planters Part II: Field performance. Comput Electron Agr, 2018; 145: 11-17.

[13] Ranta O, Drocas I, Stanila S, Molnar A, Muntean M, Marian O. The main advantages of E-Drive system used for precision seeding. Bulletin UASVM Agriculture, 2012; 69(1): 238-243.

[14] Zhang C, Wu R, Chen L. Design and test of electronic control seeding system for maize. Transactions of the CSAM, 2017; 48(2): 51-59. (in Chinese)

[15] Yang L, He X T, Cui T, Zhang D X, Shi S, Zhang R, et al. Development of mechatronic driving system for seed meters equipped on conventional precision corn planter. Int J Agric \& Biol Eng, 2015; 8(4): 1-9.

[16] Ding Y, Yang L, Zhang D X, Cui T, He X, Zhong X. Control system of motor-driving maize precision planter based on GPS speed measurement. Transactions of the CSAM, 2018; 49(8): 42-49. (in Chinese)

[17] SpeedTube. Precision Planting LLC, $2020 . \quad$ Available: https://www.precisionplanting.com/products/product/speedtube. Accessed on [2020-06-14].

[18] True Speed Meter. Kinze Manufacturing Inc., 2020. Available: https://www.kinze.com/planter-performance/meters/true-speed-meter/. Accessed on [2020-06-12].

[19] Discovering what the John Deere ExacteMerge planter can do Machinefinder $\quad$ Blog, 2019. Available: https://blog.machinefinder.com/30135/john-deere-exactemerge-planter. Accessed on [2020-06-12]. 